\title{
Anastrepha species (Diptera: Tephritidae), their hosts and parasitoids in the extreme north of Brazil
}

\author{
Alberto Luiz Marsaro Júnior ${ }^{1}$, Ricardo Adaime ${ }^{2,5}$, Beatriz Ronchi-Teles $^{3}$, \\ Camila Ribeiro Lima ${ }^{4}$ \& Paulo Roberto Valle da Silva Pereira ${ }^{I}$ \\ ${ }^{1}$ Embrapa Trigo - EMBRAPA, Rod. BR 285, Km 294, CP 451, CEP 99001-970, Passo Fundo, RS, Brazil \\ ${ }^{2}$ Embrapa Amapá - EMBRAPA, Rod. JK, Km 5, n. 2600, CEP 68903-419, Macapá, Amapá, Brazil \\ ${ }^{3}$ Instituto Nacional de Pesquisas da Amazônia - INPA, \\ Av. André Araújo, 2936, Aleixo, CEP 69060-001, Manaus, AM, Brazil \\ ${ }^{4}$ Universidade do Estado do Amapá - UEAP, \\ Av. Presidente Vargas, 650, CEP 68906-970, Macapá, AP, Brazil \\ ${ }^{5}$ Corresponding author: Ricardo Adaime, e-mail: adaime@cpafap.embrapa.br
}

MARSARO JÚNIOR, A.L., ADAIME, R., RONCHI-TELES, B., LIMA, C.R. \& PEREIRA, P.R.V.S. Anastrepha species (Diptera: Tephritidae), their hosts and parasitoids in the extreme north of Brazil. Biota Neotrop. 11(4): http://www.biotaneotropica.org.br/v11n4/en/abstract?article+bn02711042011

\begin{abstract}
This work was carried out in five municipalities of the State of Roraima, Brazil, aiming to record the occurrence of Anastrepha species, their host plants and parasitoids. Fleshy fruits of 21 species of 10 families were sampled from September/2007 to September/2008. We collected 10 species of Anastrepha (Anastrepha bahiensis Lima and Anastrepha montei Lima were reported for the first time in Roraima) and five species of parasitoids (4 Braconidae and 1 Figitidae). Doryctobracon areolatus (Szépligeti) was the most abundant parasitoid.
\end{abstract}

Keywords: fruit flies, diversity, Amazon, Anastrepha bahiensis, Anastrepha montei.

MARSARO JÚNIOR, A.L., ADAIME, R., RONCHI-TELES, B., LIMA, C.R. \& PEREIRA, P.R.V.S. Espécies de Anastrepha (Diptera: Tephritidae), seus hospedeiros e parasitoides no extremo norte do Brasil. Biota Neotrop. 11(4): http://www.biotaneotropica.org.br/v11n4/pt/abstract?article+bn02711042011

Resumo: O presente trabalho foi realizado em cinco municípios do estado de Roraima, Brasil, com o objetivo de registrar a ocorrência de espécies de Anastrepha, suas plantas hospedeiras e parasitoides. Foram coletados frutos carnosos de 21 espécies pertencentes a 10 famílias botânicas de setembro/2007 a setembro/2008. Foram identificadas 10 espécies de Anastrepha (Anastrepha bahiensis Lima e Anastrepha montei Lima foram registradas pela primeira vez em Roraima) e cinco espécies de parasitoides (4 Braconidae e 1 Figitidae). Doryctobracon areolatus (Szépligeti) foi o parasitoide mais abundante.

Palavras-chave: moscas-das-frutas, diversidade, Amazônia, Anastrepha bahiensis, Anastrepha montei. 


\section{Introduction}

Fruit flies (Diptera: Tephritidae) are some of the most relevant pest species in the world, due to their direct economic impact and the quarantine restrictions enforced by many countries in order to prevent certain species from entering their territories (Aluja \& Mangan 2008). Knowledge on the species of a given region can only be obtained by a direct survey of host fruits. This method enables the accurate association of an insect to its host plant species, unlike the more commonly used methods of collection via lures and traps (Zucchi 2000).

Fruit fly surveys, their host plants and parasitoids in the Brazilian Amazon have been intensified in recent years. The state of Roraima is located in the extreme north of the Brazilian Amazon, occupying an area of $224,298.98 \mathrm{~km}^{2}$ between latitudes $5^{\circ} 16^{\prime} \mathrm{N}$ and $1^{\circ} 25^{\prime} \mathrm{S}$; and longitudes $58^{\circ} 55^{\prime} \mathrm{W}$ and $64^{\circ} 48^{\prime} \mathrm{W}$ (Instituto... 2010a), corresponding to $2.6 \%$ of the Brazilian territory or $5.3 \%$ of the Amazonian Biome (Funcate 2006, cited by Marsaro Júnior et al. 2011). Fruit farming is a very significant source of revenue and employment in the state of Roraima. The main cultivated fruits are banana, papaya, orange, lime, pineapple, melon and watermelon (Instituto... 2010b). In addition to these crops, a wide diversity of native and exotic fruit trees is also cultivated in domestic orchards in many municipalities in the state. Many of these fruit trees are known to be or can potentially be hosts to species of Anastrepha (Marsaro Júnior et al. 2011).

The first studies on Anastrepha species occurring in the state of Roraima were conducted in the 1990s (Rafael 1991, RonchiTeles et al. 1995). Seventeen species of Anastrepha have been recorded in the region up to the present, but the host plants of six of them [Anastrepha atrigona Hendel, Anastrepha flavipennis Greene, Anastrepha hamata (Loew), Anastrepha rafaeli Norrbom \& Korytkowski, Anastrepha turpiniae Stone and Anastrepha zucchii Norrbom] remain unknown in Roraima. Six species of parasitoids have also been reported in Roraima, namely five Braconidae and one Figitidae (Marsaro Júnior et al. 2011).

The purpose of this study was to record the occurrence of Anastrepha species, their host plants and parasitoids in municipalities located in northeast Roraima, where knowledge on fruit flies was scarce.

\section{Material and Methods}

Fleshy fruits were collected between September 2007 and September 2008 in five municipalities of Roraima: Boa Vista, Bonfim, Cantá, Normandia and Pacaraima (Figure 1). The samples were collected randomly from plants that bore a good quantity of maturing or mature fruits, which were picked directly off the plant or collected from the ground (recently fallen fruits). The fruits were packed in coolboxes equipped with screens, and then taken to the Laboratório de Entomologia at Embrapa Roraima in Boa Vista. In the laboratory, the fruits were counted, weighed and arranged on plastic trays over a layer of moistened vermiculite. The trays were covered with organza cloth and fastened in place with rubber bands. The material was examined every three days. Any found puparia were removed and transferred to transparent plastic flasks containing a thin layer of moistened vermiculite. The flasks were covered with organza, fastened in place with a vented lid, then placed in climate controlled chambers under controlled temperature $\left(27 \pm 0.5^{\circ} \mathrm{C}\right)$, relative humidity $(70 \pm 10 \%)$ and photophase (12 hours).

The fruit flies and parasitoids that emerged were stored in glass flasks containing $70 \%$ ethanol and were subsequently identified with the keys published by Zucchi (2000) and Canal \& Zucchi (2000).
Voucher specimens were deposited in the collections of the Instituto Nacional de Pesquisas da Amazonia (INPA) and Embrapa Amapá.

\section{Results}

Twenty-one species of potential fruit fly hosts were sampled, ranging across 10 botanical families. A total of 5,601 fruits were collected, weighing $109.94 \mathrm{~kg}$ (Tables 1-5).

Infestation was detected in all of the sampled plant species. Ten species of Anastrepha were found, namely: Anastrepha antunesi Lima, Anastrepha bahiensis Lima, Anastrepha coronilli Carrejo \& González, Anastrepha distincta Greene, Anastrepha manihoti Lima, Anastrepha montei Lima, Anastrepha obliqua (Macquart), Anastrepha serpentina (Wiedemann), Anastrepha sororcula Zucchi and Anastrepha striata Schiner.

Five species of parasitoids were obtained: four Braconidae [(Doryctobracon areolatus (Szépligeti), Doryctobracon crawfordi (Viereck), Opius bellus Gahan and Utetes anastrephae (Viereck)] and one Figitidae [Aganaspis pelleranoi (Brèthes)].

A list of Anastrepha species, their hosts and parasitoids collected in each sampled municipality is shown below.

\section{Boa Vista}

A total of 55 samples of 10 plant species was collected, totaling 1,579 fruits $(33.4 \mathrm{~kg}$ ) (Table 1). From the 47 infested samples $(85.5 \%$ of the total), we obtained 2,643 puparia which originated individuals of four species of Anastrepha: A. obliqua, A. sororcula, A. striata and A. zenildae. The lowest and highest infestation rates were observed in rose apple ( 0.4 puparium. $\mathrm{kg}^{-1}$ ) and hog plum (245.7 puparia. $\left.\mathrm{kg}^{-1}\right)$, respectively. Guava fruits showed the highest species richness (3), with predominance of A. striata $(99.4 \%)$.

Parasitism was registered in Anastrepha larvae/pupae emerged from hog plum, jocote, umbu-cajá, Brazilian guava, guava, and Indian jujube, with the highest percentage in the last species (18.2\%) (Table 1). Two species of Braconidae (D. areolatus and $O$. bellus) and one Figitidae (A. pelleranoi) were obtained, the most abundant being $O$. bellus $(81.0 \%)$.

\section{Pacaraima}

A total of 31 samples of 10 plant species was collected, totaling 1,339 fruits $(31.2 \mathrm{~kg}$ ) (Table 2). From the 28 infested samples $(90.3 \%$ of the total), we obtained 1,742 puparia which originated individuals of nine species of Anastrepha: A. antunesi, A. bahiensis, A. distincta, A. manihoti, A. montei, A. obliqua, A. sororcula, A. serpentina and A. striata. The lowest and highest infestation rates were observed in jabuticaba (25.0 puparia. $\mathrm{kg}^{-1}$ ) and abiu (172.2 puparia. $\left.\mathrm{kg}^{-1}\right)$, respectively.

Parasitism was registered in Anastrepha larvae/pupae infesting hog plum, cassava, ice-cream-bean, Brazilian guava, Para guava, guava, and abiu (Table 2). Cassava presented the highest percentage of parasitism (38.3\%). Four species of Braconidae were obtained: $D$. areolatus, $D$. crawfordi, $O$. bellus and $U$. anastrephae. The most abundant species was $D$. areolatus $(82.2 \%)$.

\section{Bonfim}

A total of 31 samples of 10 plant species was collected, totaling 1,227 fruits $(22.06 \mathrm{~kg}$ ) (Table 3$)$. From the 26 infested samples $(83.9 \%$ of the total), we obtained 1,078 puparia from which individuals of four species of Anastrepha were recovered: A. bahiensis, A. distincta, A. obliqua and $A$. striata. The lowest and highest infestation rates were observed in cashew (0.6 puparium. $\left.\mathrm{kg}^{-1}\right)$ and hog plum (345.2 puparia. $\left.\mathrm{kg}^{-1}\right)$, respectively. 


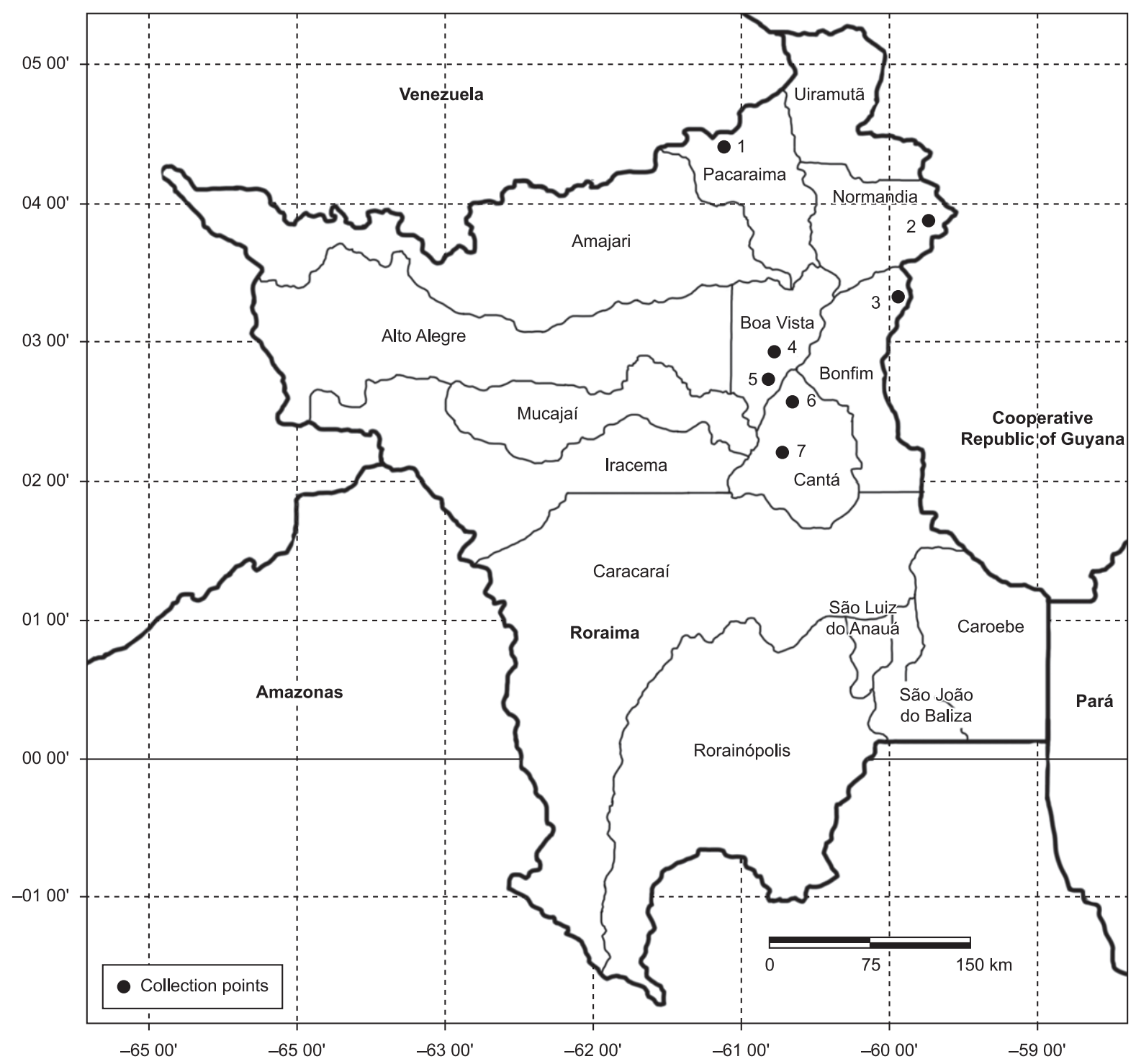

Figure 1. Map of the state of Roraima indicating the localities where Anastrepha* host fruits were collected in the five studied municipalities. *Locality 1 (Pacaraima) - Spondias mombin, Manihot esculenta, Inga edulis, Psidium sp., Psidium acutangulum, Psidium guajava, Myrciaria cauliflora, Eugenia uniflora, Eriobotrya japonica and Pouteria caimito; Locality 2 (Normandia) - S. mombin, Spondias purpurea, M. esculenta, I. edulis, Malpighia punicifolia and P. guajava; Locality 3 (Bonfim) - Anacardium occidentale, S. mombin, S. purpurea, Inga laurina, I. edulis, M. punicifolia, Psidium guineense, Psidium sp., E. uniflora and P. guajava; Locality 4 (Boa Vista) - S. purpurea, P. guineense and P. guajava; Locality 5 (Boa Vista) - S. mombin, Spondias sp., Syzygium sp., Eugenia stipitata, Averrhoa carambola and Zizyphus mauritiana; Locality 6 (Cantá) - S. mombin, S. purpurea, I. edulis, M. punicifolia and Psidium sp.; Locality 7 (Cantá) - Bellucia grossularioides and P. guajava.

Table 1. Infestation rates of several plant species by fruit flies and percentage of parasitism in Boa Vista, Roraima (September 2007 to September 2008).

\begin{tabular}{|c|c|c|c|c|c|c|c|c|}
\hline $\begin{array}{c}\text { FAMILY } \\
\text { Common name/Scientific name }\end{array}$ & $\begin{array}{l}\text { Samples } \\
\text { C/I* (n) }\end{array}$ & $\begin{array}{c}\text { Fruits } \\
\text { (n) }\end{array}$ & $\begin{array}{c}\text { Mass } \\
(\mathrm{kg})\end{array}$ & $\begin{array}{l}\text { Puparia } \\
\text { (n) }\end{array}$ & $\begin{array}{c}\text { Infestation } \\
\text { Puparia.kg }^{-1}\end{array}$ & Anastrepha spp. & $\begin{array}{c}\text { Parasitism } * * \\
(\%)\end{array}$ & Hymenoptera \\
\hline \multicolumn{9}{|l|}{ ANACARDIACEAE } \\
\hline Hog plum (Spondias mombin L.) & $10 / 10$ & 268 & 2.21 & 543 & 245.7 & $\begin{array}{c}\text { A. obliqua (127) } \\
\text { males (161) }\end{array}$ & 13.1 & $\begin{array}{c}\text { O. bellus }(70) \\
\text { A. pelleranoi }(1)\end{array}$ \\
\hline Jocote (Spondias purpurea L.) & $10 / 10$ & 396 & 3.98 & 542 & 136.2 & $\begin{array}{c}\text { A. obliqua }(136) \\
\text { males (194) }\end{array}$ & 4.1 & O. bellus (22) \\
\hline Umbu-cajá (Spondias sp.) & $1 / 1$ & 14 & 0.28 & 60 & 214.3 & $\begin{array}{l}\text { A. obliqua (30) } \\
\text { males }(15)\end{array}$ & 10.0 & O. bellus (6) \\
\hline \multicolumn{9}{|l|}{ MYRTACEAE } \\
\hline Brazilian guava (Psidium guineense $\mathrm{Sw}$.) & $5 / 5$ & 43 & 1.31 & 156 & 119.1 & $\begin{array}{l}\text { A. striata }(31) \\
\text { males }(68)\end{array}$ & 2.6 & D. areolatus (4) \\
\hline
\end{tabular}

*C/I: Collected/Infested (other data expressed in the table refer exclusively to infested samples). 
Table 1. Continued...

\begin{tabular}{|c|c|c|c|c|c|c|c|c|}
\hline $\begin{array}{c}\text { FAMILY } \\
\text { Common name/Scientific name } \\
\end{array}$ & $\begin{array}{l}\text { Samples } \\
\text { C/I* (n) }\end{array}$ & $\begin{array}{c}\text { Fruits } \\
\text { (n) }\end{array}$ & $\begin{array}{c}\text { Mass } \\
(\mathbf{k g})\end{array}$ & $\begin{array}{l}\text { Puparia } \\
\text { (n) }\end{array}$ & $\begin{array}{c}\text { Infestation } \\
\text { Puparia.kg }^{-1} \\
\end{array}$ & Anastrepha spp. & $\begin{array}{c}\text { Parasitism** } \\
(\%) \\
\end{array}$ & Hymenoptera \\
\hline Araza (Eugenia stipitata Mc Vaugh) & $5 / 4$ & 42 & 4.25 & 169 & 39.8 & $\begin{array}{l}\text { A. obliqua }(70) \\
\text { males }(65)\end{array}$ & 0 & - \\
\hline Guava (Psidium guajava L.) & $10 / 10$ & 232 & 11.35 & 994 & 87.6 & $\begin{array}{l}\text { A. striata }(343) \\
\text { A. obliqua }(1) \\
\text { A. zenildae }(1) \\
\text { males (399) }\end{array}$ & 0.8 & D. areolatus (8) \\
\hline Pitanga (Eugenia uniflora L.) & $4 / 3$ & 219 & 0.59 & 94 & 159.3 & $\begin{array}{l}\text { A. obliqua }(25) \\
\text { males }(25)\end{array}$ & 0 & - \\
\hline Rose apple (Syzygium sp.) & $2 / 1$ & 26 & 2.55 & 1 & 0.4 & $\begin{array}{c}\text { A. sororcula }(1) \\
\text { males }(0)\end{array}$ & 0 & - \\
\hline \multicolumn{9}{|l|}{ OXALIDACEAE } \\
\hline Star fruit (Averrhoa carambola L.) & $4 / 1$ & 61 & 4.22 & 29 & 6.9 & $\begin{array}{l}\text { A. obliqua (2) } \\
\text { males (3) }\end{array}$ & 0 & - \\
\hline \multicolumn{9}{|l|}{ RHAMNACEAE } \\
\hline Indian jujube (Zizyphus mauritiana Lam.) & $4 / 2$ & 278 & 2.66 & 55 & 20.7 & $\begin{array}{l}\text { A. zenildae (19) } \\
\text { males (16) }\end{array}$ & 18.2 & D. areolatus (10) \\
\hline
\end{tabular}

Table 2. Infestation rates of several plant species by fruit flies and percentage of parasitism in Pacaraima, Roraima (September 2007 to September 2008).

\begin{tabular}{|c|c|c|c|c|c|c|c|c|}
\hline \multirow{2}{*}{$\begin{array}{c}\text { FAMILY } \\
\text { Common name/Scientific name }\end{array}$} & \multirow{2}{*}{$\begin{array}{l}\text { Samples } \\
\text { C/I* (n) }\end{array}$} & \multirow{2}{*}{$\begin{array}{l}\text { Fruits } \\
\text { (n) }\end{array}$} & \multirow{2}{*}{$\begin{array}{c}\text { Mass } \\
(\mathrm{kg})\end{array}$} & \multirow{2}{*}{$\begin{array}{l}\text { Puparia } \\
\text { (n) }\end{array}$} & \multirow{2}{*}{$\frac{\text { Infestation }}{\text { Puparia.kg }^{-1}}$} & \multirow{2}{*}{ Anastrepha spp. } & \multirow{2}{*}{$\begin{array}{c}\text { Parasitism** } \\
(\%)\end{array}$} & \multirow{2}{*}{ Hymenoptera } \\
\hline & & & & & & & & \\
\hline ANACARDIACEAE & & & & & & & & \\
\hline Hog plum (Spondias mombin L.) & $2 / 2$ & 85 & 1.11 & 150 & 135.1 & $\begin{array}{l}\text { A. obliqua (44) } \\
\text { A. antunesi (2) } \\
\text { males (43) }\end{array}$ & 7.3 & $\begin{array}{c}\text { O. bellus (10) } \\
\text { U. anastrephae (1) }\end{array}$ \\
\hline \multicolumn{9}{|l|}{ EUPHORBIACEAE } \\
\hline Cassava (Manihot esculenta Crantz) & $2 / 2$ & 596 & 1.19 & 133 & 111.8 & $\begin{array}{l}\text { A. manihoti }(26) \\
\text { A. montei }(1) \\
\text { males }(36)\end{array}$ & 38.3 & D. areolatus (51) \\
\hline FABACEAE & & & & & & & & \\
\hline Ice-cream-bean (Inga edulis Mart.) & $4 / 4$ & 50 & 8.96 & 326 & 36.4 & $\begin{array}{l}\text { A. distincta }(148) \\
\text { males (89) }\end{array}$ & 0.9 & D. areolatus (3) \\
\hline \multicolumn{9}{|l|}{ MYRTACEAE } \\
\hline Brazilian guava (Psidium sp.) & $2 / 2$ & 71 & 1.86 & 125 & 67.2 & $\begin{array}{l}\text { A. striata }(32) \\
\text { A. obliqua (4) } \\
\text { males (37) }\end{array}$ & 19.2 & D. areolatus (24) \\
\hline Para guava (Psidium acutangulum DC.) & $2 / 2$ & 49 & 4.34 & 109 & 25.1 & $\begin{array}{l}\text { A. striata (17) } \\
\text { A. obliqua (22) } \\
\text { males (39) }\end{array}$ & 0.92 & D. areolatus (1) \\
\hline Guava (Psidium guajava L.) & $10 / 10$ & 222 & 10.47 & 443 & 42.3 & $\begin{array}{c}\text { A. striata }(133) \\
\text { males }(163)\end{array}$ & 3.4 & D. areolatus (15) \\
\hline Jabuticaba [Myrciaria cauliflora (DC.) O. Berg] & $1 / 1$ & 61 & 0.36 & 9 & 25.0 & $\begin{array}{l}\text { A. obliqua (2) } \\
\text { A. sororcula }(1) \\
\text { males (3) }\end{array}$ & 0 & - \\
\hline Pitanga (Eugenia uniflora L.) & $4 / 2$ & 162 & 0.48 & 37 & 77.1 & $\begin{array}{l}\text { A. obliqua (12) } \\
\text { males (14) }\end{array}$ & 0 & - \\
\hline \multicolumn{9}{|l|}{ ROSACEAE } \\
\hline Loquat [Eriobotrya japonica (Thunb.) Lindl.] & $2 / 1$ & 7 & 0.09 & 7 & 77.8 & $\begin{array}{c}\text { A. bahiensis (1) } \\
\text { males (1) }\end{array}$ & 0 & - \\
\hline \multicolumn{9}{|l|}{ SAPOTACEAE } \\
\hline Abiu [Pouteria caimito (Ruiz \& Pav.) Radlk. ] & $2 / 2$ & 36 & 2.34 & 403 & 172.2 & $\begin{array}{l}\text { A. serpentina }(144) \\
\text { males }(131)\end{array}$ & 7.4 & $\begin{array}{l}\text { D. areolatus }(17) \\
\text { D. crawfordi }(13)\end{array}$ \\
\hline
\end{tabular}

*C/I: Collected/Infested (other data expressed in the table refer exclusively to infested samples). 
Parasitism was registered in Anastrepha larvae/pupae infesting hog plum, Brazilian guava (Psidium guineense), guava and jocote (Table 3). The highest percentage of parasitism was observed in Anastrepha larvae/pupae infesting hog plum (10.7\%), from which three species of Braconidae were obtained: $O$. bellus, D. areolatus and $U$. anastrephae. Doryctobracon areolatus accounted for $52.6 \%$ of collected parasitoids.

\section{Normandia}

A total of 14 samples of 6 plant species was collected, totaling 696 fruits $(16.27 \mathrm{~kg}$ ) (Table 4). All samples were infested, yielding 351 puparia from which individuals of four species of Anastrepha emerged: A. distincta, A. manihoti, A. obliqua and A. striata. The lowest and highest infestation rates were observed in ice-cream-bean $\left(8.8\right.$ puparia. $\left.\mathrm{kg}^{-1}\right)$ and hog plum $\left(140.0\right.$ puparia. $\left.\mathrm{kg}^{-1}\right)$, respectively.

Table 3. Infestation rates of several plant species by fruit flies and percentage of parasitism in Bonfim, Roraima (September 2007 to September 2008).

\begin{tabular}{|c|c|c|c|c|c|c|c|c|}
\hline $\begin{array}{c}\text { FAMILY } \\
\text { Common name/Scientific name }\end{array}$ & $\begin{array}{l}\text { Samples } \\
\text { C/I* (n) }\end{array}$ & $\begin{array}{l}\text { Fruits } \\
\text { (n) }\end{array}$ & $\begin{array}{c}\text { Mass } \\
(\mathrm{kg})\end{array}$ & $\begin{array}{l}\text { Puparia } \\
\text { (n) }\end{array}$ & $\frac{\text { Infestation }}{\text { Puparia.kg }^{-1}}$ & Anastrepha spp. & $\begin{array}{c}\text { Parasitism*** } \\
(\%)\end{array}$ & Hymenoptera \\
\hline \multicolumn{9}{|l|}{ ANACARDIACEAE } \\
\hline Cashew (Anacardium occidentale L.) & $3 / 1$ & 27 & 3.09 & 2 & 0.6 & $\begin{array}{l}\text { A. obliqua }(1) \\
\text { males (1) }\end{array}$ & 0 & 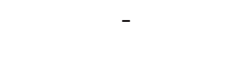 \\
\hline Hog plum (Spondias mombin L.) & $4 / 4$ & 90 & 0.73 & 252 & 345.2 & $\begin{array}{l}\text { A. obliqua (58) } \\
\text { males }(62)\end{array}$ & 10.7 & $\begin{array}{l}\text { O. bellus }(24) \\
\text { D. areolatus }(2)\end{array}$ \\
\hline Jocote (Spondias purpurea L.) & $1 / 1$ & 84 & 0.86 & 104 & 120.9 & $\begin{array}{l}\text { A. obliqua (28) } \\
\text { males (41) }\end{array}$ & 4.8 & $\begin{array}{c}\text { U. anastrephae (1) } \\
\text { D. areolatus (3) } \\
\text { U. anastrephae (2) }\end{array}$ \\
\hline \multicolumn{9}{|l|}{ FABACEAE } \\
\hline Sacky sac bean [Inga laurina (Sw.) Willd.] & $1 / 1$ & 39 & 0.77 & 7 & 9.1 & $\begin{array}{c}\text { A. distincta }(5) \\
\text { males }(0)\end{array}$ & 0 & - \\
\hline Ice-cream-bean (Inga edulis Mart.) & $2 / 2$ & 60 & 10.20 & 20 & 2.0 & $\begin{array}{l}\text { A. distincta }(8) \\
\text { males (2) }\end{array}$ & 0 & - \\
\hline \multicolumn{9}{|l|}{ MALPIGHIACEAE } \\
\hline Acerola (Malpighia punicifolia L.) & $6 / 3$ & 564 & 1.83 & 14 & 7.7 & $\begin{array}{l}\text { A. obliqua (7) } \\
\text { males (3) }\end{array}$ & 0 & - \\
\hline \multicolumn{9}{|l|}{ MYRTACEAE } \\
\hline Brazilian guava (Psidium guineense Sw.) & $6 / 6$ & 156 & 1.86 & 337 & 181.2 & $\begin{array}{l}\text { A. striata (78) } \\
\text { A. bahiensis (1) } \\
\text { males (101) }\end{array}$ & 3.3 & D. areolatus (1) \\
\hline Brazilian guava (Psidium sp.) & $1 / 1$ & 74 & 0.26 & 40 & 153.8 & $\begin{array}{l}\text { A. striata }(10) \\
\text { males }(15)\end{array}$ & 0 & - \\
\hline Guava (Psidium guajava L.) & $6 / 6$ & 47 & 2.46 & 292 & 118.7 & $\begin{array}{l}\text { A. striata }(74) \\
\text { males }(128)\end{array}$ & 8.2 & D. areolatus (24) \\
\hline Pitanga (Eugenia uniflora L.) & $1 / 1$ & 86 & 0.31 & 10 & 32.3 & $\begin{array}{l}\text { A. obliqua (4) } \\
\text { males (4) }\end{array}$ & 0 & \\
\hline
\end{tabular}

*C/I: Collected/Infested (other data expressed in the table refer exclusively to infested samples).

Table 4. Infestation rates of several plant species by fruit flies and percentage of parasitism in Normandia, Roraima (September 2007 to September 2008).

\begin{tabular}{|c|c|c|c|c|c|c|c|c|}
\hline $\begin{array}{c}\text { FAMILY } \\
\text { Common name/Scientific name }\end{array}$ & $\begin{array}{l}\text { Samples } \\
\text { C/I* (n) }\end{array}$ & $\begin{array}{l}\text { Fruits } \\
\text { (n) }\end{array}$ & $\begin{array}{c}\text { Mass } \\
(\mathbf{k g})\end{array}$ & $\begin{array}{l}\text { Puparia } \\
\text { (n) }\end{array}$ & $\frac{\text { Infestation }}{\text { Puparia.kg }^{-1}}$ & Anastrepha spp. & $\begin{array}{l}\text { asitism } * * \\
(\%)\end{array}$ & Hymenoptera \\
\hline \multicolumn{9}{|l|}{ ANACARDIACEAE } \\
\hline Hog plum (Spondias mombin L.) & $1 / 1$ & 10 & 0.05 & 7 & 140.0 & $\begin{array}{l}\text { A. obliqua }(2) \\
\text { males }(1)\end{array}$ & 14.3 & D. areolatus (1) \\
\hline Jocote (Spondias purpurea L.) & $3 / 3$ & 190 & 1.84 & 119 & 64.7 & $\begin{array}{l}\text { A. obliqua }(13) \\
\text { males }(17)\end{array}$ & 46.2 & $\begin{array}{c}\text { D. areolatus (48) } \\
\text { U. anastrephae (6) } \\
\text { O. bellus (1) }\end{array}$ \\
\hline \multicolumn{9}{|l|}{ EUPHORBIACEAE } \\
\hline Cassava (Manihot esculenta Crantz) & $1 / 1$ & 155 & 0.33 & 7 & 21.2 & $\begin{array}{l}\text { A. manihoti }(4) \\
\text { males }(2)\end{array}$ & 0 & - \\
\hline \multicolumn{9}{|l|}{ FABACEAE } \\
\hline Ice-cream-bean (Inga edulis Mart.) & $3 / 3$ & 33 & 8.38 & 74 & 8.8 & $\begin{array}{l}\text { A. distincta }(25) \\
\text { males (20) }\end{array}$ & 0 & - \\
\hline \multicolumn{9}{|l|}{ MALPIGHIACEAE } \\
\hline Acerola (Malpighia punicifolia L.) & $2 / 2$ & 229 & 0.83 & 12 & 14.5 & $\begin{array}{l}\text { A. obliqua }(5) \\
\text { males }(5)\end{array}$ & 8.3 & D. areolatus (1) \\
\hline $\begin{array}{l}\text { MYRTACEAE } \\
\text { Guava (Psidium guajava L.) }\end{array}$ & $4 / 4$ & 79 & 4.84 & 132 & 27.3 & $\begin{array}{l}\text { A. striata }(50) \\
\text { males }(42)\end{array}$ & 3.0 & D. areolatus (4) \\
\hline
\end{tabular}

*C/I: Collected/Infested (other data expressed in the table refer exclusively to infested samples). 
Parasitism was registered in hog plum, jocote, acerola, and guava (Table 4). Jocote had the highest percentage of parasitism (46.2\%). Three species of Braconidae were obtained: D. areolatus, O. bellus and $U$. anastrephae. Doryctobracon areolatus accounted for $88.5 \%$ of collected parasitoids.

\section{Cantá}

A total of 11 samples of 7 plant species was collected, totaling 760 fruits $(6.7 \mathrm{~kg}$ ) (Table 5). All samples were infested, yielding 438 puparia from which individuals of four species of Anastrepha were recovered: A. coronilli, A. distincta, A. obliqua and A. striata. The lowest and highest infestation rates were observed in ice-creambean (1.3 puparium $/ \mathrm{kg}$ ) and jocote (291.2 puparia/kg), respectively.

Parasitism was registered in Anastrepha larvae/pupae infesting hog plum, jocote, acerola, goiaba-de-anta ("tapir guava"), and guava (Table 4). Larvae infesting Brazilian guava showed the highest percentage of parasitism (16.7\%). Two species of Braconidae were obtained: D. areolatus $(48.5 \%)$ and $O$. bellus $(51.5 \%)$.

\section{Discussion}

Among the 10 species of Anastrepha obtained in this study, two (A. bahiensis and A. montei) are first records in the state of Roraima.

Anastrepha bahiensis was obtained in loquat (Eriobotrya japonica) in Pacaraima (Table 2) and in Brazilian guava (Psidium guineense) in Bonfim (Table 3). Ten host species of A. bahiensis are known, belonging to the families Moraceae (4 species), Myrtaceae (4), Annonaceae (1), and Ulmaceae (1) (Zucchi 2008, Zucchi et al. 2011a). This work is the first report of loquat and Brazilian guava as hosts of A. bahiensis in Brazil. This record of A. bahiensis in Pacaraima and Bonfim expands the distribution of this species in Brazil, which previously ranged across eight states including only two in the northern region (Amapá and Amazonas).

Anastrepha montei was collected from cassava (Manihot esculenta) in Pacaraima (Table 2). This species had already been found in 11 Brazilian states. This is the second time that A. montei is reported in the Brazilian Northern region, as it had already been found in the state of Tocantins (Zucchi 2008).

In addition to loquat (Eriobotrya japonica) and Brazilian guava (Psidium guineense), other seven plant species are listed for the first time as hosts of Anastrepha in Roraima: umbu-cajá (Spondias sp.), rose apple (Syzygium sp.), star fruit (Averrhoa carambola L.), Brazilian guava (Psidium sp.), jabuticaba (Myrciaria cauliflora), cashew (Anacardium occidentale L.), and sacky sac bean (Inga laurina).

This study also provided a better understanding of the geographic distribution of Anastrepha species in the sampled area. First records of species were made in the municipalities of Boa Vista (A. sororcula), Pacaraima (A. antunesi, A. bahiensis, A. manihoti, A. montei, and A. sororcula), Bonfim (A. bahiensis), and Normandia (A. manihoti). In the municipality of Cantá, all of the observed species are reported for the first time at the location (A. coronilli, A. distincta, A. obliqua, and A. striata).

Anacardiaceae showed the highest infestation rates. In hog plum, infestation ranged from 135.1 puparia. $\mathrm{kg}^{-1}$ in Pacaraima to 345.2 puparia. $\mathrm{kg}^{-1}$ in Bonfim (Tables 2 and 3). In jocote, infestation ranged from 64.7 puparia. $\mathrm{kg}^{-1}$ in Normandia to 291.2 puparia. $\mathrm{kg}^{-1}$ in Cantá (Tables 4 and 5). Interestingly, an even higher infestation rate (532.6 puparia. $\mathrm{kg}^{-1}$ ) had already been obtained in a sample of hog plum collected in Amajari, Roraima (Marsaro Júnior et al. 2010). These rates are high if compared to the highest value already observed in the state of Amapá (141.8 puparia. $\mathrm{kg}^{-1}$ ), where a significant number of samples has already been assessed (Silva et al. 2011).

Anastrepha obliqua and A. striata were the species with the largest number of host plant. Anastrepha obliqua was associated to 12 plant species (6 Myrtaceae, 4 Anacardiaceae, 1 Malpighiaceae, and 1 Oxalidaceae) and A. striata to five (4 Myrtaceae and 1 Anacardiaceae).

Regarding parasitoids, all recorded species had already been reported in Roraima. The most recently reported was $D$. crawfordi (Zucchi et al. 2011b). Out of the 407 parasitoid specimens observed in this study, $57.2 \%$ were $D$. areolatus, the parasitoid most widely distributed in Brazil (Leonel Junior et al. 1995, Canal \& Zucchi 2000) and Latin America (Ovruski et al. 2000). Doryctobracon areolatus was the most abundant parasitoid species in the municipalities of Pacaraima (Table 2) and Normandia (Table 4). In the municipalities of Bonfim (Table 3) and Cantá (Table 5), the populations of D. areolatus and $O$. bellus were almost equally abundant. In Boa Vista, O. bellus was the most abundant species $(81.0 \%)$ (Table 1$)$. In species of Anacardiaceae, $O$. bellus was predominant and was sometimes the

Table 5. Infestation rates of several plant species by fruit flies and percentage of parasitism in Cantá, Roraima (September 2007 to September 2008).

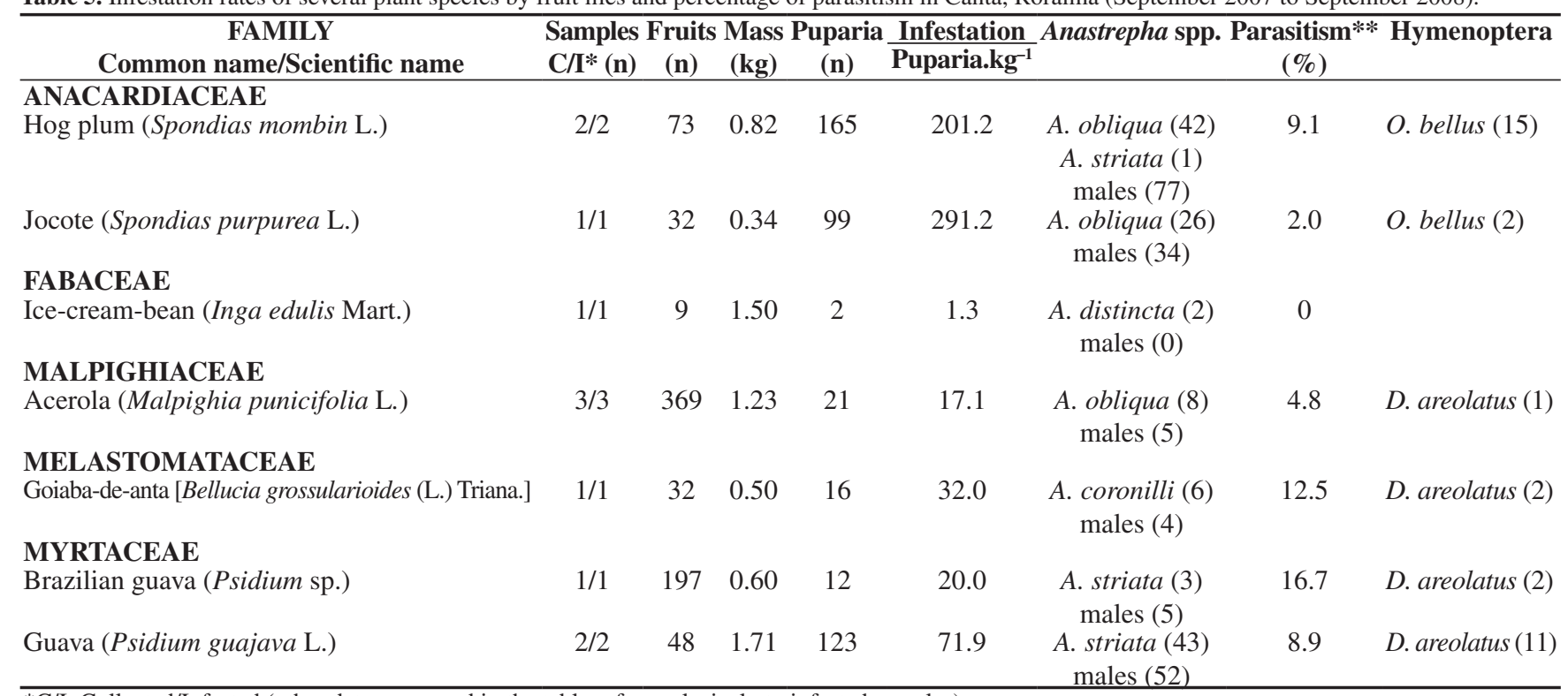


only species found (Tables 1-3 and 5). Specifically in hog plum, $95.2 \%$ of observed parasitoid individuals were $O$. bellus. The remainder belonged to three species: A. pelleranoi, $U$. anastrephae and $D$. areolatus. The parasitism rates in hog plum ranged from $7.3 \%$ in Pacaraima (Table 2) to $14.3 \%$ in Normandia (Table 4). The species is therefore important as a natural reservoir of parasitoids.

\section{Conclusions}

Based on fruit samplings performed in municipalities in the northeast region of the state of Roraima, Brazil, it is possible to conclude that:

- Anastrepha obliqua is the most abundant and polyphagous species;

- Hog plum presents the highest rates of infestation by Anastrepha;

- Doryctobracon areolatus and Opius bellus are the most abundant parasitoid species.

\section{Acknowledgements}

To the CNPq for the Research Productivity Fellowships granted to RA. To biologist Wilson Rodrigues da Silva for identifying part of the Anastrepha specimens. To Prof. Dr. Roberto Antonio Zucchi for identification of Doryctobracon crawfordi. To Dr. Jorge Anderson Guimarães for identification of Aganaspis pelleranoi (Brèthes).

\section{References}

ALUJA, M. \& MANGAN, R. 2008. Fruit fly (Diptera: Tephritidae) host status determination: critical conceptual, methodological, and regulatory considerations. Annu Rev Entomol. 53:473-502. PMid:17877455. http:// dx.doi.org/10.1146/annurev.ento.53.103106.093350

CANAL, N.A. \& ZUCCHI, R.A. 2000. Parasitóides - Braconidae. In Moscas-das-frutas de importância econômica no Brasil: conhecimento básico e aplicado (A. Malavasi \& R.A. Zucchi, Ed.). Holos, Ribeirão Preto, p.119-126.

INSTITUTO BRASILEIRO DE GEOGRAFIA E ESTATÍSTICA IBGE. 2010a. Roraima - Síntese. http://www.ibge.gov.br/estadosat/perfil. php?sigla=rr (último acesso em 21/06/2010).

INSTITUTO BRASILEIRO DE GEOGRAFIA E ESTATÍSTICA IBGE. 2010b. Produção agrícola municipal: informações sobre culturas temporárias e permanentes. http://www.sidra.ibge.gov.br/bda/pesquisas/ pam/default.asp (último acesso em 27/03/2010).
LEONEL JUNIOR, F.L., ZUCCHI, R.A. \& WHARTON, R.A. 1995. Distribution and tephritid hosts (Diptera) of braconid parasitoids (Hymenoptera) in Brazil. Int. J. Pest Manag. 41:208-213. http://dx.doi. org/10.1080/09670879509371951

MARSARO JÚNIOR, A.L., RONCHI-TELES, B., BARBOSA, R.I., SILVA JÚNIOR, R.J., AGUIAR, R.M. \& SILVA, R.A. 2011. Conhecimento sobre moscas-das-frutas no Estado de Roraima. In Moscas-das-frutas na Amazônia brasileira: diversidade, hospedeiros e inimigos naturais (R.A. Silva, W.P. Lemos \& R.A. Zucchi, eds.). Embrapa Amapá, Macapá, p.279-290.

MARSARO JÚNIOR, A.L., SILVA, R.A., SILVA, W.R., LIMA, C.R., FLORES, A.S. \& RONCHI-TELES, B. 2010. New records of Anastrepha (Diptera: Tephritidae), its hosts and parasitoids in the Serra do Tepequém, Roraima state, Brazil. Rev. Agric. 85(1):15-19.

OVRUSKI, S.M., ALUJA, M., SIVINSKI, J. \& WHARTON, R.A. 2000. Hymenopteran parasitoids on fruit-infesting Tephritidae (Diptera) in Latin America and the Southern United States: diversity, distribution, taxonomic status and their use in fruit fly biological control. Integr. Pest Manag. Rev. 5(2):81-107. http://dx.doi.org/10.1023/A:1009652431251

RAFAEL, J.A. 1991. Insetos coletados durante o Projeto Maracá, Roraima, Brasil: lista complementar. Acta Amazon. 21(4):325-336.

RONCHI-TELES, B., ZUCCHI, R.A. \& SILVA, N.M. 1995. Novos registros de espécies de Anastrepha (Dip. Tephritidae) e seus hospedeiros no Estado de Roraima. In Congresso Brasileiro de Entomologia. Sociedade Entomológica do Brasil, Caxambu, p.239.

SILVA, R.A., DEUS, E.G., PEREIRA, J.D.B., JESUS, C.R., SOUZA-FILHO, M.F. \& ZUCCHI, R.A. 2011. Conhecimento sobre moscas-das-frutas no Estado do Amapá. In Moscas-das-frutas na Amazônia brasileira: diversidade, hospedeiros e inimigos naturais (R.A. Silva, W.P. Lemos \& R.A. Zucchi, eds.). Embrapa Amapá, Macapá, p.223-236.

ZUCCHI, R.A. 2000. Taxonomia. In Moscas-das-frutas de importância econômica no Brasil: conhecimento básico e aplicado (A. Malavasi \& R.A. Zucchi, eds.). Holos, Ribeirão Preto, p.13-24.

ZUCCHI, R.A. 2008. Fruit flies in Brazil - Anastrepha species and their host plants. Esalq/USP, Piracicaba. www.lea.esalq.usp.br/anastrepha/ (último acesso em 11/05/2011).

ZUCCHI, R.A., DEUS, E.G. \& SILVA, R.A. 2011a. Espécies de Anastrepha e seus hospedeiros na Amazônia brasileira. In Moscas-das-frutas na Amazônia brasileira: diversidade, hospedeiros e inimigos naturais (R.A. Silva, W.P. Lemos \& R.A. Zucchi, eds.). Embrapa Amapá, Macapá, p. 51-70.

ZUCCHI, R.A., MARINHO, C.F \& SILVA, R.A. 2011b. First record of the fruit fly parasitoid Doryctobracon crawfordi (Viereck) (Hym., Braconidae) in Brazil. Neotrop. Entomol. 40(6): 711-712. 
